\title{
Linear and Nonlinear Features of Few-mode Fibers with Partial Coupling among Groups of Quasi-degenerate Modes
}

\author{
Filipe M. Ferreira \\ Aston Institute of Photonic Technologies, School of Engineering and Applied Science, Aston University, Birmingham, UK \\ from Nov. 2019 with Optical Networks Group, Dept. of Electronic \& Electrical Engineering, University College London, UK \\ filipef@ieee.org
}

\begin{abstract}
We review different solution methods for the linear coupling operator in the coupled nonlinear Schrödinger equations for few-mode propagation. Models are compared for different differential mode delay and linear coupling regimes. () 2020 The Author.

OCIS codes: (060.2330) Fiber optics communications; (060.4370) Nonlinear optics, fibers.
\end{abstract}

\begin{abstract}
1. Introduction
Spatial-division multiplexing (SDM) has emerged as one possible solution to overcome the capacity limit of singlemode fibers (SMFs) [1]. Among the different SDM approaches, mode-division multiplexing (MDM) over few-mode fibers (FMFs) offers the highest spatial information density. However, the multitude of spatial modes introduces additional linear and nonlinear impairments, namely: group delay (GD) spread [2-4] given the interplay between differential mode delay (DMD) and linear mode coupling (LMC), intermodal nonlinear effects (IM-NL) [5-7], and mode dependent loss (MDL) [8, 9]. Chief among them is LMC that plays a crucial role at controlling the GD spread, MDL accumulation and the efficiency of the overall nonlinear interactions. Thereby, and with practical fibers operating in all LMC regimes, the modelling of LMC has been under intensive research [9-13] as well as its impact on the statistics of GD, MDL and NL. Here we review some of the most representative LMC models proposed for transmission in FMFs covering all coupling regimes but with special emphasis in partial coupling between groups of quasi-degenerate modes - also known as intermediate coupling regime. Transmission simulations for wavelengthdivision-multiplexed (WDM) MDM systems demonstrate the application scope of the different LMC models.
\end{abstract}

\section{Linear Mode Coupling and Nonlinear Transmission Modelling}

Three LMC regimes are usually identified: weak coupling (WC), strong coupling (SC) and intermediate coupling [13]. WC usually refers to the case of 2 (or more) non-degenerate mode groups whose inter-group coupling can be neglected, likely in (multiple-)step-index or elliptical core FMFs. Intermediate coupling generally applies to FMFs supporting several non-degenerate mode groups whose inter-group coupling cannot be neglected for the transmission distance of interest, likely in graded-core fibers optimized for low DMD [2]. SC usually applies to the coupling between (quasi-)degenerate modes within a given mode group of a FMF, or the coupling between closely spaced cores in multi-core fibers (MCFs) [4], or simply the coupling between the two polarization modes in a SMF. Quantitatively, the LMC regime in which a fiber link is operating can be determined given the coupling length or the coupling strength; figures of merit originally proposed for polarization mode dispersion in SMFs that can be extended for SDM fibers. The coupling strength (XT) can be quantified as the ratio between the average power in all the other orthogonal modes and the average power remaining in the launching mode $(m)$ after a certain distance, $X T=\sum_{v \neq \mathrm{m}}\left(P_{v} / P_{m}\right)$, where $m$ is the mode showing higher coupling strength. And, the coupling length $\left(L_{c}\right)$ can be defined as the length for which the average power in the orthogonal modes is within $e^{-2}$ of the power in the launching mode, this is equivalent to $X T=\left[e^{2}-1\right] /\left[e^{2}+1\right](-1.18 \mathrm{~dB})$. Finally, a fiber link is said to operate in the SC-regime if the transmission distance is much longer than $L_{c}$, and vice-versa for the WC-regime.

Nonlinear transmission modelling in SDM fibers involves solving the coupled nonlinear Schrödinger equation (CNLSE) in [5-7]. Its numerical integration can be achieved considering three CNLSE operators, dispersion, LMC and nonlinearity, acting independently for a sufficiently short integration step. In such case, the LMC operator can be resolved in two ways: numerically, having to generate random coupling matrices every step with a given coupling strength; analytically via new Manakov equations derived by averaging the nonlinear operator over all possible LMC realizations. And, in the numerical approach there are 2 main variants referred here as: lumped LMC and distributed LMC. In the following, numerical and analytical handlings of LMC in nonlinear transmission are reviewed.

\section{$2.1 \quad$ Lumped LMC Modelling}

In this case, multi-section modelling includes LMC among non-degenerate modes via random unitary matrices with a section length just longer than $L_{c}$ such that accumulated $X T$ equals $0 \mathrm{~dB}$ in average [3]. This approach is very convenient in the linear power regime allowing matching the analytical predictions for GD statistics [3] provided that $L_{c}$ is much shorter than the dispersion length $T_{0}{ }^{2} /\left|\beta_{u}{ }^{(2)}\right|$ and the walk-off length $T_{0} /\left|\beta_{u}{ }^{(1)}-\beta_{v}{ }^{(1)}\right| ; \beta_{m}{ }^{(l)}$ is the $l^{\text {th }}$ order 
coefficient of a Taylor series expansion of mode $m$ propagation constant; $T_{0}$ is the bandwidth reciprocal. In the nonlinear power regime, there is an additional requirement to the applicability of the lumped LMC model, $L_{c}$ must be much shorter than the nonlinear effective length $L_{e f f}=[1-\exp (-\alpha L)] / \alpha$, where $\alpha$ is the attenuation coefficient and $L$ is the span length $\left(L_{\text {eff }} \sim 20 \mathrm{~km}\right)$. This limitation makes the lumped LMC model inapplicable for many SDM fibers that operate in the weak-to-intermediate LMC regime achieving full mode mixing beyond $10 \mathrm{~km}$. Nevertheless, this model is still applicable to SDM fibers in the WC-regime since LMC between non-degenerate can be neglected.

\subsection{Distributed LMC Modelling}

A semi-analytical model capable of describing the LMC for fibers operating in the intermediate coupling regime has been proposed by the author in $[10,13]$. In this model all LMC is assumed to arise from core-cladding imperfections which are discretized by dividing the fiber in multiple sections, each with a random displacement of the core center position. This simplification allows deriving semi-analytical solutions of the LMC operator that can be used in the numerical integration of the CNLSE. The LMC strength is set using a fixed amount of radial displacement and a random azimuth displacement given by a uniform distribution. In this way, it was shown in [13] that at each step a random amount of LMC is introduced among non-degenerate modes that in average approximates the desired level. This method has been proven accurate in the linear power regime, matching the analytical GD statistics in FMF links for transmission lengths up to $10,000 \mathrm{~km}$, in any LMC regime, with and without GD management [13]. In Section 3, the applicability of this model to nonlinear propagation in SDM fibers is reviewed.

\subsection{Manakov Equations}

Manakov equations have been derived for the WC- and SC-regimes by averaging the CNLSE over all possible occurrences of the LMC operator. In the SC-regime, the averaging considers random full coupling between all guided modes [6]; for a fiber guiding $2 M$ polarization modes, one single degeneracy factor emerges $4 / 3 \cdot(2 M) /(2 M+1)$, while the nonlinear parameter is averaged over all pair combinations of modes. Such operation assumes that all modes propagate with similar GD (this is, $D M D$ should not be higher than a few $\mathrm{ps} / \mathrm{km}$ ), which is the case for degenerate modes in FMFs or supermodes in coupled-core MCFs [4]. In the WC-regime, the CNLSE is averaged considering randomly coupled modes within each mode group and neglecting inter-group coupling [7], the intra-group degeneracy factor becomes $4 / 3 \cdot\left(2 M_{u}\right) /\left(2 M_{u}+1\right)$, for a group with $2 M_{u}$ polarization modes, and the nonlinear parameter is averaged over all pair combinations in the group, similarly, the inter-group degeneracy factor becomes $4 / 3$ and the nonlinear parameter is averaged over all mixed pair combinations (one mode from each group). The Manakov equations for the SC-regime may provide a significant integration time advantage, since that in the distributed LMC approach the average $X T$ per step should be $\leq-20 \mathrm{~dB}$, and this does not apply to Manakov. Otherwise, the generalized Manakov equations do not provide any significant advantage in terms of the integration time required for numerical solutions as discussed in section IV of [14].

\section{Results and Discussion}

This section reviews the author recent comparison of the models discussed above for full system simulation [15], this is: WC-Manakov, SC-Manakov, distributed LMC, and lumped LMC. A symmetric implementation of the splitstep Fourier method is used to solve the CNLSE, the step size is adapted to keep the local error smaller than $10^{-5}$ (smaller values led to negligible change). A MDM system using a FMF with 6 linearly polarized (LP) modes (LP $\mathrm{P}_{01}$, $\mathrm{LP}_{02}, \mathrm{LP}_{11 \mathrm{a}}, \mathrm{LP}_{11 \mathrm{~b}}, \mathrm{LP}_{21 \mathrm{a}}$ and $\mathrm{LP}_{21 \mathrm{~b}}$ ) each with 2 orthogonal polarisations is considered, full fiber details in [16]. $672 \mathrm{Gbit} / \mathrm{s}$ per channel are transmitted in an optical super-channel with multiple WDM channels (per mode) modulated with 14 Gbaud polarisation-multiplexed 16QAM, $14.1 \mathrm{GHz}$ spaced. The simulations here consider $2^{16}$ symbols per polarisation mode and $2^{11}$ training symbols. For nonlinear dominated performance $20 \mathrm{~km}$ spans with $3 \mathrm{~dB}$ noise figure amplifiers are used. Additional details on the simulation setup, channel estimation and equalization in [15]. The figure of merit in the following is the minimum $Q$-factor among the 12 polarization modes of the center WDM channel, estimated using the mean and standard deviation of the received symbols.

Fig. 1 shows the $Q$-factor as a function of $X T$ for $-2 \mathrm{dBm} /$ channel (i.e. in the nonlinear regime) in the absence of $D M D$ for different numbers of WDM channels over several spans of $20 \mathrm{~km}$ : 3 channels over 25 spans, 5 channels over 21 spans and 11 channels over 15 spans. These channel\&span combinations were selected to obtain roughly the same performance in the WC-regime. The results in Fig. 1 confirm the agreement between all models in the extreme LMC regimes for all channel\&span combinations. However, in the intermediate LMC regime $(-70 \mathrm{~dB} / \mathrm{m}$ to $-30 \mathrm{~dB} / \mathrm{m})$ the lumped LMC and the distributed LMC models are found to be in qualitative agreement but not quantitative; the Manakov approximations are not applicable in this regime. Importantly, the $Q$-factor results show that performance degrades with $X T$ before it finally improves when approaching the SC-regime. As $X T$ increases, from WC-regime to the intermediate LMC regime, additional phase rotations introduced by LMC allow intermodal four-wave-mixing phase matching to be achieved for a broader range of frequency combinations than it would be possible in the absence of LMC, therefore degrading performance since there is no significant averaging of the nonlinear 


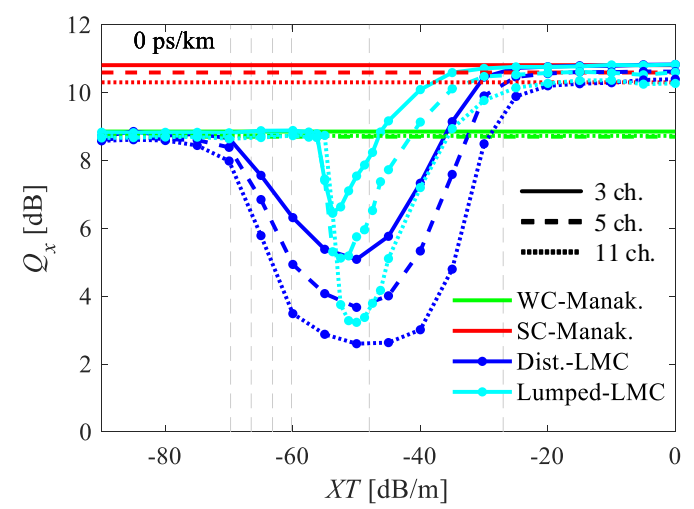

Fig. 1. $Q$-factor as a function of $X T$ at a launch power of $-2 \mathrm{dBm} / \mathrm{ch}$, no $D M D$, for: 3 channels over 25 spans, 5 channels over 21 spans, and 11 channels over 15 spans. Data points averaged over 10 repetitions. Dashed vertical lines identify the fibers studied in [15].

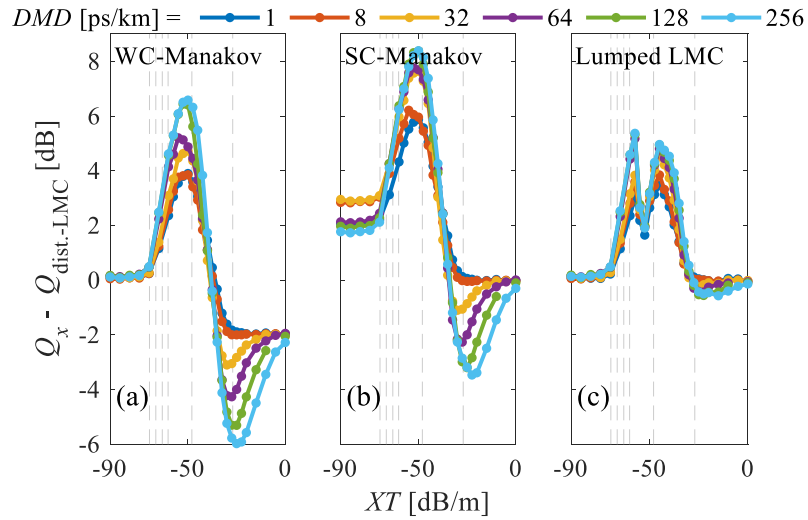

Fig. 2. $Q$-factor error as a function of $X T$ using the distributed $L M C$ model as reference (-2 $\mathrm{dBm} / \mathrm{ch}, 3$ channels and 25 spans) for different models: (a) WCManakov, (b) SC-Manakov, and (c) lumped LMC. Data points averaged over 10 repetitions. Dashed vertical lines identify the fibers studied in [15].

coefficients. A broader range of frequency combinations means that the additional nonlinear penalty grows significantly with the number of WDM channels, as the results in Fig. 1 confirm. Finally, by increasing XT towards the SC-regime, fast random rotations of the hyper-polarization state of the field along the fibre reduce the efficiency of the overall nonlinear process, averaging the nonlinear coefficients, improving performance.

To further evaluate the applicability of the different methods, Fig. 2 shows $Q$-factor error (taking the distributed LMC model as reference) as a function of $X T$ for a wide range of $D M D$ values, 1-to- $256 \mathrm{ps} / \mathrm{km}$. In Fig. 2-(a), for extremely small $X T$ values $(<-70 \mathrm{~dB} / \mathrm{m})$, WC-Manakov generates accurate results even for $D M D$ as high as $256 \mathrm{ps} / \mathrm{km}$, thus mostly relevant for uncouple-core MCFs as practical FMFs have $X T \geq-70 \mathrm{~dB} / \mathrm{m}$. In Fig. 2-(b), for high $X T$ values $(>-30 \mathrm{~dB} / \mathrm{m})$, SC-Manakov is only accurate if $D M D<10 \mathrm{ps} / \mathrm{km}$, thereby mainly applicable to coupled-core MCFs [4]. From Fig. 2-(c), the lumped LMC model is able to accurately model propagation for $X T>-30 \mathrm{~dB} / \mathrm{m}$ even for $D M D$ over $100 \mathrm{ps} / \mathrm{km}$, thus widely applicable to the coupled-core MCFs presented in literature [4]. Finally, in the intermediate coupling regime $(-70 \mathrm{~dB} / \mathrm{m}$ to $-30 \mathrm{~dB} / \mathrm{m})$ only a distributed LMC model capable of introducing controllable LMC over small step-sizes (e.g. $0.1-100 \mathrm{~m})$ can accurately model transmission.

\section{Conclusions}

Fibers operating in the intermediate coupling regime $(-70 \mathrm{~dB} / \mathrm{m}$ to $-30 \mathrm{~dB} / \mathrm{m})$ have worse performance than fibers in the WC-regime, thus it should be avoided when attempting to minimise the nonlinear noise penalty. The best operating regime is the SC-regime as it reduces the nonlinear penalty. However with many fibres operating at the edges of the extreme coupling regimes, the distributed LMC model is an essential tool to develop future SDM systems.

I would like to thank Associate Professor Sygletos Stylianos, Professor Andrew Ellis and Professor Nick Doran from Aston University for their continued support. This work has been funded by the Engineering and Physical Sciences Research Council (Grants EP/R024057/1-FPA-ROCS, EP/M005283/1-UPON, EP/L000091/1-PEACE, EP/R035342/1TRANSNET). To access the underlying data see https://doi.org/10.17036/researchdata.aston.ac.uk.00000436.

\section{References}

[1] D. J. Richardson, et al.,, "Space-division multiplexing in optical fibres," Nature Photonics 7, 354-362 (2013).

[2] L. Gruner-Nielsen et al., "Few Mode Transmission Fiber With Low DGD, Low Mode Coupling, and Low Loss," J Lightw Technol 30, $3693-3698$ (2012).

[3] K.-P. Ho and J. Kahn, "Linear Propagation Effects in Mode-Division Multiplexing Systems," J Lightw Technol 32, 614-628 (2014).

[4] T. Hayashi, et al.,, "Record-Low Spatial Mode Dispersion and Ultra-Low Loss Coupled Multi-Core Fiber for Ultra-Long-Haul Transmission," J Lightwave Technol 35, 450-457 (2017).

[5] F. Ferreira, et al., "Nonlinear semi-analytical model for simulation of few-mode fiber transmission," Photon Technol Lett 24, 240-242 (2012).

[6] A. Mecozzi, et al., "Nonlinear propagation in multi-mode fibers in the strong coupling regime," Opt Express 20, 11673-11678 (2012).

[7] A. Mecozzi, et al.,"Coupled Manakov equations in multimode fibers with strongly coupled groups of modes," Opt Express 20, 23436-441 (2012).

[8] K.-P. Ho and J. Kahn, "Mode-dependent loss and gain: statistics and effect on mode-division multiplexing," Opt Express 19, 16612-16635 (2011).

[9] C. Antonelli, et al., "Modeling and performance metrics of MIMO-SDM systems with different amplification schemes in the presence of modedependent loss," Opt Express 23, 2203-2219 (2015).

[10] F. Ferreira, et al., "Semi-analytical model for linear modal coupling in few-mode fiber transmission," in ICTON 2012, p. Th.A1.5.

[11] C. Antonelli, et al., "The delay spread in fibers for SDM transmission: dependence on fiber parameters and perturbations," Opt Express 23, 2196-2202 (2015).

[12] A. Mecozzi, et al., "Intensity impulse response of SDM links," Opt Express 23, 5738-43 (2015).

[13] F. Ferreira, et al., "Semi-Analytical Modelling of Linear Mode Coupling in Few-Mode Fibers," J Lightw Technol 35, 4011-4022 (2017).

[14] C. Antonelli, et al., "Modeling of Nonlinear Propagation in Space-Division Multiplexed Fiber-Optic Transmission," J Light Technol 34, 36-54 (2016).

[15] F. Ferreira, et al., "Nonlinear Performance of Few-Mode Fiber Links With Intermediate Coupling," J Lightw Technol 37, 989-999 (2019).

[16] F. Ferreira, et al., "Design of Few-Mode Fibers With M-modes and Low Differential Mode Delay," J Lightw Technol 32, 353-360 (2014). 